\title{
Complete Query Answering Over Horn Ontologies Using a Triple Store
}

\author{
Yujiao Zhou, Yavor Nenov, Bernardo Cuenca Grau, and Ian Horrocks \\ Department of Computer Science, University of Oxford, UK
}

\begin{abstract}
In our previous work, we showed how a scalable OWL 2 RL reasoner can be used to compute both lower and upper bound query answers over very large datasets and arbitrary OWL 2 ontologies. However, when these bounds do not coincide, there still remain a number of possible answer tuples whose status is not determined. In this paper, we show how in the case of Horn ontologies one can exploit the lower and upper bounds computed by the RL reasoner to efficiently identify a subset of the data and ontology that is large enough to resolve the status of these tuples, yet small enough so that the status can be computed using a fullyfledged OWL 2 reasoner. The resulting hybrid approach has enabled us to compute exact answers to queries over datasets and ontologies where previously only approximate query answering was possible.
\end{abstract}

\section{Introduction}

An increasing number of applications rely on RDF and SPARQL for storing and querying semistructured data. The functionality of many such applications is enhanced by an OWL 2 ontology, which is used to (i) unambiguously specify the meaning of data in the application, (ii) provide the vocabulary and background knowledge needed for users to formulate accurate queries, and (iii) enrich query answers with information not explicitly represented in the dataset.

However, the appealing benefits of using an OWL 2 ontology come at the cost of scalability, since answering queries over OWL 2 ontologies is of very high computational complexity. Despite intensive efforts at optimisation, fully-fledged OWL 2 reasoners, such as HermiT [16], Pellet [23] and Racer [9], still fall far short of meeting the scalability demands of applications that require efficient management of large-scale RDF datasets.

To achieve more favourable scalability, a common approach is to delegate reasoning and query answering tasks to a rule-based RDF triple store. Stateof-the-art triple stores such as OWLim [3], Oracle's RDF Semantic Graph [26] and RDFox 1 provide robust and scalable query answering support for ontologies in the OWL 2 RL profile [17] and datasets containing millions, or even billions, of triples. However, such triple stores are intrinsically limited in their reasoning capabilities, as they ignore (parts of) axioms in the application's ontology that

\footnotetext{
1 http://www.cs.ox.ac.uk/isg/tools/RDFox/
} 
aren't captured by OWL 2 RL. As a result, they are incomplete: for some combinations of ontology, query and data, they will fail to return all query answers.

In this paper, we propose a novel approach to query answering that addresses the scalability challenge for ontology languages beyond OWL 2 RL without giving up completeness of query answers. The key idea is to employ a hybrid technique that combines an OWL 2 RL reasoner based on a highly scalable RDF triple store (RL reasoner for short) with a fully-fledged OWL 2 reasoner (OWL reasoner for short) such that most of the computational workload can be delegated to the RL reasoner, and the OWL reasoner is used only as necessary to ensure completeness. The difficulty in realising this approach is to efficiently determine when and where fully-fledged reasoning is needed.

Our hybrid query answering technique builds on previous work [27], where we showed how an RL reasoner can be exploited to efficiently compute lower and upper bound query answers over very large datasets and arbitrary OWL 2 ontologies. When the two bounds coincide (which was often the case in the experiments reported in [27]), the query has been fully answered. When the two bounds do not coincide, however, there may still remain a significant number of possible answer tuples whose status is undetermined. In theory, the status of these tuples can be determined using an OWL reasoner, but for large-scale datasets, even checking single tuples is often infeasible in practice.

The main contribution of this paper is a technique for identifying a (typically small) subset of the dataset and ontology that is sufficient for determining the status of a possible answer tuple. The basic idea is that, starting from a query $Q$ and a possible answer tuple $\vec{a}$, we use backward chaining with axioms from the upper-bound ontology to identify those axioms and data triples from the input ontology and dataset that might contribute to a proof that $\vec{a}$ is an answer to $Q$. An OWL reasoner is then used to check if the identified axioms and data triples entail that $\vec{a}$ is an answer to $Q$. Currently, our technique is only known to be applicable to Horn ontologies (i.e., ontologies that can be translated to first-order Horn clauses). However, many OWL 2 ontologies are Horn 6, as are all the profiles. Moreover, we conjecture that the approach can be extended to arbitrary OWL 2 ontologies; verifying this conjecture is left for future work.

Our new technique also addresses an important limitation with the approach presented in 27: if the upper-bound ontology and dataset is unsatisfiable, then it is necessary to check the satisfiability of the input ontology and dataset, but this was impractical with large datasets. Now, we can simply use our new technique to compute the answer to the query owl:Nothing $(x)$, with the ontology and dataset being satisfiable iff the answer is empty.

We have developed a reasoner that integrates RDFox and the HermiT OWL reasoner. A preliminary evaluation has shown very promising results. For instance, we can compute in reasonable time the exact answers to a range of queries over the LUBM(40) ontology and dataset-results that are far beyond the capabilities of any other OWL reasoner known to us. Our technique appears to be very effective in identifying small relevant subsets of the data and ontology: for many queries, only $2 \%$ of the data and just a few axioms from the ontology 
were necessary to determine the status of all unverified answer tuples. Moreover, the effectiveness of our technique is not restricted to LUBM: we have obtained encouraging results with the Fly Anatomy ontology - a biomedical ontology containing more than 7,000 classes and 140,000 axioms - and its associated dataset.

\section{Preliminaries}

We adopt standard notions from first-order logic with equality, such as variables, constants, terms, atoms, formulas, sentences, substitutions, entailment (written $=)$, and (un)satisfiability. The equality atom between terms $t$ and $t^{\prime}$ is denoted as $t \approx t^{\prime}$; we use the abbreviation $t \not \approx t^{\prime}$ for $\neg\left(t \approx t^{\prime}\right)$ (an inequality atom). The falsum atom is denoted as $\perp$ (equivalent to owl: Nothing), whereas the dual universal truth atom is denoted as $T$ (equivalent to owl: Thing).

OWL 2 Ontologies. We assume familiarity with the normative specifications of OWL 2 and OWL 2 RL. We deviate slightly from the normative documents only in that we make an explicit distinction between schema-level and data-level axioms. We use ontology and dataset to refer to a set of schema-level and a set of data-level axioms, respectively. W.l.o.g. we assume that data assertions are given as facts (ground atoms), each of which corresponds to a single RDF triple. Consider as our running example the following ontology $\mathcal{O}^{e x}$ and dataset $\mathcal{D}^{e x}$.

$$
\begin{aligned}
\mathcal{O}^{e x}=\{ & \text { SubClassOf }(\text { Animal SomeValuesFrom }(\text { eats Thing })), \\
& \text { SubClassOf }(\text { Herbivore AllValuesFrom (eats Plant })), \\
& \text { DisjointClasses(Herbivore Carnivore }), \\
& \text { SubClassOf }(\text { Carnivore MinCardinality }(2 \text { hasParent Thing })))\} \\
\mathcal{D}^{e x}=\{ & \text { ClassAssertion (Animal lion }), \\
& \text { ClassAssertion (Animal rabbit }), \\
& \text { ClassAssertion (Herbivore rabbit }), \\
& \text { ClassAssertion (Herbivore sheep }), \\
& \text { PropertyAssertion(eats sheep grass }), \\
& \text { ClassAssertion (Carnivore wolf })\}
\end{aligned}
$$

Queries. A conjunctive query (CQ) is a first-order formula in the form of $Q(\vec{x})=$ $\exists \vec{y}(\varphi(\vec{x}, \vec{y}))$ with $Q$ a distinguished query predicate and $\varphi(\vec{x}, \vec{y})$ a conjunction of atoms without inequalities. The variables in $\vec{x}$ are distinguished. The following CQ with a distinguished variables $x$ asks for all individuals that eat plants:

$$
Q^{e x}(x):=\exists y(\operatorname{eats}(x, y) \wedge \operatorname{Plant}(y)) .
$$

A tuple of constants $\vec{a}$ is a certain answer to $Q(\vec{x})$ w.r.t. a set of first-order sentences $\mathcal{F}$ and a set of facts $\mathcal{D}$ if $\mathcal{F} \cup \mathcal{D}=Q(\vec{a})$. The set of all certain answers to $Q(\vec{x})$ w.r.t. $\mathcal{F}$ and $\mathcal{D}$ is denoted as $\operatorname{cert}(Q, \mathcal{F}, \mathcal{D})$. For example, the individuals sheep and rabbit are certain answers to $Q^{e x}$ w.r.t. $\mathcal{O}^{e x}$ and $\mathcal{D}^{e x}$. We omit the 
distinguished variables of $Q(\vec{x})$ and write just $Q$ for brevity. SPARQL conjunctive queries are CQs with only distinguished variables 2

Rule languages. Rule languages are widely-used knowledge representation formalisms that have strong connections with different fragments of OWL 2 [4]. Specifically, OWL 2 RL is strongly connected to datalog, whereas Horn ontologies are related to datalog ${ }^{ \pm}$- an extension of datalog with existential quantifiers allowed in rule heads. For instance, our example ontology $\mathcal{O}^{e x}$ is equivalent to the following datalog ${ }^{ \pm}$rules in which all free variables are universally quantified.

$$
\begin{aligned}
& \exists y(\text { eats }(x, y)) \leftarrow \operatorname{Animal}(x) \\
& \text { Plant }(y) \leftarrow \operatorname{eats}(x, y) \wedge \operatorname{Herbivore}(x) \\
& \perp \leftarrow \text { Carnivore }(x) \wedge \operatorname{Herbivore}(x) \\
& \exists y_{1} \exists y_{2}\left(\text { hasParent }\left(x, y_{1}\right) \wedge \text { hasParent }\left(x, y_{2}\right) \wedge y_{1} \not y_{2}\right) \leftarrow \text { Carnivore }(x)
\end{aligned}
$$

Formally, a datalog ${ }^{ \pm}$rule is a first-order sentence of the following form [5]:

$$
\forall \vec{x}\left(\exists \vec{y}\left(C_{1} \wedge \cdots \wedge C_{m}\right) \leftarrow B_{1} \wedge \cdots \wedge B_{n}\right),
$$

where each $B_{j}$ is an atom with variables in $\vec{x}$ that is neither $\perp$ nor an inequality atom, and either (i) $m=1$ and $C_{1}=\perp$, or (ii) $m \geq 1$ and, for each $1 \leq i \leq m$, $C_{i}$ is an atom different from $\perp$ with free variables in $\vec{x} \cup \vec{y}$. A datalog rule is a rule of the form (1) with no existentially quantified variables ${ }^{3}$ A datalog (resp. datalog $^{ \pm}$) program is a set of datalog (resp. datalog ${ }^{ \pm}$) rules.

Horn ontologies (i.e., ontologies that can be normalised as a set of first-order Horn clauses) can also be represented by datalog ${ }^{ \pm}$programs. Furthermore, each OWL 2 RL ontology can be represented by a datalog program. Axioms T2 and T3 in our running example are in OWL $2 \mathrm{RL}$ and can be represented by the datalog rules $\mathrm{P} 2$ and P3 respectively; in contrast, T1 is outside OWL 2 RL and, as such, is only expressible by a datalog ${ }^{ \pm}$rule (in our case P1).

Datalog rules allow for easy and efficient computation of the dataset $\mathcal{D}_{\Sigma}$ consisting of all facts entailed by a datalog program $\Sigma$ and a dataset $\mathcal{D}$. The $\operatorname{set} \mathcal{D}_{\Sigma}$ is called the materialisation of $\Sigma$ w.r.t. $\mathcal{D}$. The set of certain answers $\operatorname{cert}(Q, \Sigma, \mathcal{D})$ for an arbitrary query $Q$ coincides with $\operatorname{cert}\left(Q, \emptyset, \mathcal{D}_{\Sigma}\right)$. Consider, for example, the set $\Sigma_{L}$ comprising the datalog rules $\mathrm{P} 2$ and $\mathrm{P} 3$. The materialisation of $\Sigma_{L}$ w.r.t. $\mathcal{D}^{e x}$ extends $\mathcal{D}^{e x}$ with the single fact ClassAssertion(Plant grass); clearly, sheep is an answer to the query $Q^{e x}$ w.r.t. $\Sigma_{L}$ and $\mathcal{D}^{e x}$, but rabbit is not.

\section{Our Approach in a Nutshell}

In this paper, we propose a hybrid reasoning technique that combines an RL reasoner based on a highly scalable RDF triple store with a fully-fledged OWL

\footnotetext{
${ }^{2}$ In general, a SPARQL query can include non-distinguished variables; however, the semantics of SPARQL means that this is equivalent to treating all variables as distinguished and then applying a suitable projection.

${ }^{3}$ Our definition of datalog is slightly non-standard as it allows conjunction in rule heads; such rules can be equivalently split into multiple rules with atomic heads.
} 


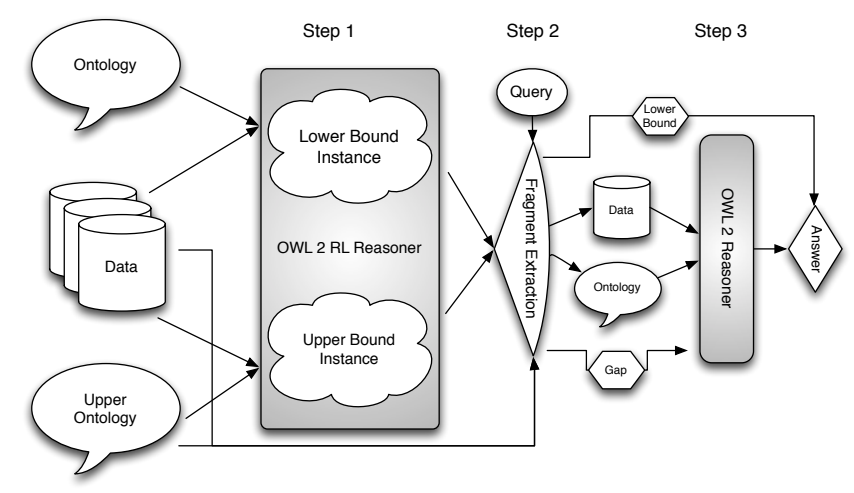

Fig. 1. Overview

reasoner. The key feature of our query answering technique is that it tries to delegate most of the computational workload to the RL reasoner, thus minimising the use of the less scalable OWL reasoner. Given a Horn OWL 2 ontology $\mathcal{O}$, a dataset $\mathcal{D}$, and a CQ $Q$, we compute the certain answers cert $(Q, \mathcal{O}, \mathcal{D})$ in three steps, which we summarise next and schematically depict in Figure 1

Step 1: Lower and upper bound query answers. Our first step is to compute two OWL $2 \mathrm{RL}$ ontologies $\mathcal{O}_{L}$ (the lower bound ontology) and $\mathcal{O}_{U}$ (the upper bound ontology) satisfying the following property: $\operatorname{cert}\left(Q, \mathcal{O}_{L}, \mathcal{D}\right) \subseteq$ $\operatorname{cert}(Q, \mathcal{O}, \mathcal{D}) \subseteq \operatorname{cert}\left(Q, \mathcal{O}_{U}, \mathcal{D}\right)$. Since both $\mathcal{O}_{L}$ and $\mathcal{O}_{U}$ are OWL 2 RL ontologies, we can then use an RL reasoner to compute the lower bound cert $\left(Q, \mathcal{O}_{L}, \mathcal{D}\right)$ and the upper bound $\operatorname{cert}\left(Q, \mathcal{O}_{U}, \mathcal{D}\right)$. If the set $G=\operatorname{cert}\left(Q, \mathcal{O}_{U}, \mathcal{D}\right) \backslash \operatorname{cert}\left(Q, \mathcal{O}_{L}, \mathcal{D}\right)$ of tuples in the "gap" between lower and upper bound is empty, then the set of certain answers cert $(Q, \mathcal{O}, \mathcal{D})$ coincides with both lower and upper bounds, in which case we don't need to resort to the OWL reasoner. This step exploits the techniques in our previous work [27, which we briefly recapitulate in Section 4 .

Step 2: Computing ontology and dataset fragments. In the second step we exploit the lower and upper bound ontologies and query answers to identify (small) fragments $\mathcal{O}_{f}$ of $\mathcal{O}$ and $\mathcal{D}_{f}$ of $\mathcal{D}$ satisfying: $\mathcal{O} \cup \mathcal{D} \models Q(\vec{a})$ iff $\mathcal{O}_{f} \cup \mathcal{D}_{f}=$ $Q(\vec{a})$ for each $\vec{a} \in G$. Thus, $\mathcal{O}_{f}$ and $\mathcal{D}_{f}$ are sufficient for determining whether each tuple in $G$ is indeed a certain answer to $Q$. The fragments $\mathcal{O}_{f}$ and $\mathcal{D}_{f}$ depend on both the input query $Q$ and the tuples in $G$. This novel technique is the main contribution of our paper, and it is described in Section 5 .

Step 3: Calling the OWL reasoner. In the final step we resort to the OWL reasoner to verify whether $\mathcal{O}_{f} \cup \mathcal{D}_{f}=Q(\vec{a})$ for each tuple $\vec{a} \in G$. We return as certain answers the union of the lower bound and the verified tuples in $G$ : $\operatorname{cert}(Q, \mathcal{O}, \mathcal{D})=\operatorname{cert}\left(Q, \mathcal{O}_{L}, \mathcal{D}\right) \cup\left\{\vec{a} \in G \mid \mathcal{O}_{f} \cup \mathcal{D}_{f}=Q(\vec{a})\right\}$.

\section{Lower and Upper Bound Query Answers}

In our previous work [27] we showed how an RL reasoner can be used to efficiently compute upper and lower bound query answers over arbitrary OWL 2 ontologies. 
In this section, we recapitulate the techniques proposed there. Our description will be rather informal, and we refer the interested reader to [27] for details.

Lower bound answers. RL reasoners are flexible enough to process arbitrary ontologies on a "best efforts" basis; that is, the reasoner ignores (parts of) the axioms that are outside OWL 2 RL, thus effectively reasoning with a lower bound ontology $\mathcal{O}_{L}$. RL reasoners are guaranteed to be sound (i.e., $\mathcal{O} \models \mathcal{O}_{L}$ ), and hence all the tuples they compute are indeed certain answers; we can therefore compute lower bound answers simply by running the RL reasoner as a "black box" on the input $Q, \mathcal{O}$, and $\mathcal{D}$. For instance, when given our example ontology $\mathcal{O}^{e x}$, dataset $\mathcal{D}^{e x}$, and query $Q^{e x}$, a typical RL reasoner will reduce $\mathcal{O}^{e x}$ to the OWL 2 RL ontology $\mathcal{O}_{L}^{e x}=\left\{\begin{array}{|l|l|l|}T 2 & T 3\end{array}\right.$, and will compute $\operatorname{cert}\left(Q^{e x}, \mathcal{O}_{L}^{e x}, \mathcal{D}^{e x}\right)=\{$ sheep $\}$.

Upper bound answers. We transform $\mathcal{O}$ into an OWL 2 RL ontology $\mathcal{O}_{U}$ such that $\mathcal{O}_{U} \models \mathcal{O}$. First, $\mathcal{O}$ is normalised into a datalog ${ }^{ \pm}$program $\Sigma^{ \pm}$using a variant of the structural transformation of first-order logic (see [16 27]). For instance, our example ontology $\mathcal{O}^{e x}$ can be normalised into the datalog ${ }^{ \pm}$program consisting of rules P1 P4. The crucial second step is the transformation of the resulting datalog ${ }^{ \pm}$program into a (stronger) datalog program $\Sigma_{U}$ satisfying $\Sigma_{U}=\mathcal{O}$; roughly speaking, $\Sigma_{U}$ is obtained by Skolemising all existential quantifiers into fresh constants. For example, the datalog ${ }^{ \pm}$rules $\mathrm{P} 1$ and $\mathrm{P} 4$ get transformed into the rules D1, D4 and D5 to give the following datalog program:

$$
\begin{aligned}
\Sigma_{U}^{e x}=\{ & \text { eats }(x, c) \leftarrow \operatorname{Animal}(x), \\
& \operatorname{Plant}(y) \leftarrow \operatorname{eats}(x, y) \wedge \operatorname{Herbivore}(x), \\
& \perp \leftarrow \operatorname{Carnivore}(x) \wedge \operatorname{Herbivore}(x), \\
& \text { hasParent }\left(x, c_{1}\right) \wedge \operatorname{hasParent}\left(x, c_{2}\right) \leftarrow \text { Carnivore }(x), \\
& \left.\perp \leftarrow c_{1} \approx c_{2}\right\} .
\end{aligned}
$$

Finally, the datalog program $\Sigma_{U}$ is transformed into the upper bound OWL 2 RL ontology $\mathcal{O}_{U}$, where $\mathcal{O}_{U}=\Sigma_{U}$; roughly speaking, each rule in $\Sigma_{U}$ is transformed into an OWL 2 RL axiom by "rolling up" the rule's body and head into class descriptions, while possibly introducing fresh predicates in order to satisfy the syntactic restrictions of OWL 2 RL. For instance, the datalog rules D1,D5 in our running example are transformed into the following OWL 2 RL axioms:

$$
\begin{aligned}
\mathcal{O}_{U}^{e x}=\{ & \text { SubClassOf }(\text { Animal Has Value }(\text { eats } c)), \\
& \text { SubClassOf }(\text { Herbivore AllValuesFrom }(\text { eats Plant })), \\
& \text { DisjointClasses }(\text { Herbivore Carnivore }), \\
& \text { SubClassOf }\left(\text { Carnivore Has Value }\left(\text { hasParent } c_{1}\right)\right), \\
& \text { SubClassOf }\left(\text { Carnivore HasValue }\left(\text { hasParent } c_{2}\right)\right), \\
& \text { DifferentFrom } \left.\left(c_{1} c_{2}\right)\right\} .
\end{aligned}
$$

As a result, we obtain that $\mathcal{O}_{U} \models \mathcal{O}$ and hence $\operatorname{cert}(Q, \mathcal{O}, \mathcal{D}) \subseteq \operatorname{cert}\left(Q, \mathcal{O}_{U}, \mathcal{D}\right)$. Clearly, the transformation of $\mathcal{O}$ into the upper bound ontology $\mathcal{O}_{U}$ will in general introduce consequences that are not entailed by the original ontology $\mathcal{O}$. To 
see this, consider again our running example. The axioms R1, R2, A1 and A2 entail ObjectPropertyAssertion (eats rabbit c), ObjectPropertyAssertion (eats lion c) and ClassAssertion(Plant $c$ ). We thus get that (in addition to sheep) rabbit and lion are also answers to $Q^{e x}$, i.e. $\operatorname{cert}\left(Q^{e x}, \mathcal{O}_{U}^{e x}, \mathcal{D}^{e x}\right)=\{$ sheep, rabbit, lion $\}$. However, we have that lion $\notin \operatorname{cert}\left(Q^{e x}, \mathcal{O}^{e x}, \mathcal{D}^{e x}\right)$.

The final transformation from $\Sigma_{U}$ into $\mathcal{O}_{U}$ is only required if the RL reasoner to be used only accepts OWL 2 RL ontologies; our RL reasoner RDFox can handle datalog rules natively, and this transformation can be dispensed with.

Dealing with unsatisfiability. An important limitation with the approach presented in 27] is that, given an ontology $\mathcal{O}$ and a dataset $\mathcal{D}$, if $\mathcal{O}_{L} \cup \mathcal{D}$ is satisfiable (i.e., $\operatorname{cert}\left(\perp(x), \mathcal{O}_{L}, \mathcal{D}\right)=\emptyset$ ) but $\mathcal{O}_{U} \cup \mathcal{D}$ is unsatisfiable (i.e., $\operatorname{cert}\left(\perp(x), \mathcal{O}_{U}, \mathcal{D}\right) \neq \emptyset$ ), we must check if $\mathcal{O} \cup \mathcal{D}$ is satisfiable; if $\mathcal{O} \cup \mathcal{D}$ is satisfiable, we can still use the above procedure to compute upper bound answers, but if $\mathcal{O} \cup \mathcal{D}$ is not satisfiable, then everything is entailed and the (upper bound) answer to any query is trivially the set of all tuples of the appropriate arity that can be formed from individuals in $\mathcal{D}$. The difficulty is that, when $\mathcal{D}$ is large, it may be impractical to check the satisfiability of $\mathcal{O} \cup \mathcal{D}$ using an OWL reasoner.

We can now address this issue by using our new hybrid query answering technique: if $\operatorname{cert}\left(\perp(x), \mathcal{O}_{L}, \mathcal{D}\right)=\emptyset$, but $\operatorname{cert}\left(\perp(x), \mathcal{O}_{U}, \mathcal{D}\right) \neq \emptyset$, then in Step 2 we will compute fragments $\mathcal{O}_{f}$ and $\mathcal{D}_{f}$ for $\perp(x)$, and in Step 3 we will use these fragments with an OWL reasoner to compute cert $(\perp(x), \mathcal{O}, \mathcal{D})$. Clearly, $\mathcal{O} \cup \mathcal{D}$ is satisfiable iff $\operatorname{cert}(\perp(x), \mathcal{O}, \mathcal{D})=\emptyset$.

\section{Computing Ontology and Dataset Fragments}

Given an input ontology $\mathcal{O}$, a dataset $\mathcal{D}$ and a set of possible answer tuples $G$, our goal is to compute small ontology $\mathcal{O}_{f} \subseteq \mathcal{O}$ and dataset $\mathcal{D}_{f} \subseteq \mathcal{D}$ such that $\mathcal{O}_{f} \cup \mathcal{D}_{f} \models Q(\vec{a})$ iff $\mathcal{O} \cup \mathcal{D} \models Q(\vec{a})$ for each tuple $\vec{a} \in G$.

\subsection{Overview}

In a nutshell, our technique for computing $\mathcal{O}_{f}$ and $\mathcal{D}_{f}$ works as follows.

1. We consider the upper bound datalog rules $\Sigma_{U}$ and, for each $\vec{a} \in G$, we compute all (minimal) proofs of $Q(\vec{a})$ in $\Sigma_{U} \cup \mathcal{D}$. Specifically, we consider "backward chaining" proofs based on SLD-resolution.

2. We define $\mathcal{D}_{f}$ (resp. $\Sigma_{f}$ ) as the set of facts in $\mathcal{D}$ (resp. rules in $\Sigma_{U}$ ) that have been used in some SLD-resolution proof for some $\vec{a} \in G$.

3. Finally, we "trace back" the rules in $\Sigma_{f} \subseteq \Sigma_{U}$ to the OWL 2 axioms $\mathcal{O}_{f} \subseteq \mathcal{O}$ from which they were derived.

To illustrate this process, let us consider our example ontology $\mathcal{O}^{e x}$, data set $\mathcal{D}^{e x}$ and query $Q^{e x}$. In this case, we have $\{$ sheep $\}$ as the lower bound answer and $\{$ sheep, rabbit,lion $\}$ as the upper bound answer; our goal is thus to determine whether rabbit and lion are indeed certain answers. To this end, we consider the upper bound datalog program $\Sigma_{U}^{e x}$, and inspect all the "backward chaining" 
proofs for $Q^{e x}\left(\right.$ rabbit) and $Q^{e x}($ lion $)$ in $\Sigma_{U}^{e x} \cup \mathcal{D}^{e x}$. Consider for example the following proof of $Q^{e x}$ (rabbit).

$$
\begin{aligned}
& S_{0}:=\operatorname{eats}(\text { rabbit }, y) \wedge \operatorname{Plant}(y) \\
& S_{1}:=\operatorname{Plant}(c) \wedge \operatorname{Animal}(\text { rabbit }) \quad \text { via D1 } \\
& S_{2}:=\operatorname{Animal}(\text { rabbit }) \wedge \text { eats }(x, c) \wedge \operatorname{Herbivore}(x) \quad \text { via D2 } \\
& S_{3}:=\operatorname{eats}(x, c) \wedge \text { Herbivore }(x) \quad \text { via } A 2 \\
& S_{4}:=\operatorname{Herbivore}(x) \wedge \operatorname{Animal}(x) \quad \text { via } D 1 \\
& S_{5}:=\text { Animal }(\text { rabbit }) \quad \text { via } A 3 \\
& S_{6}:=\top \quad \text { via } A 2
\end{aligned}
$$

Starting from the goal $Q^{e x}($ rabbit $)=\operatorname{eats}($ rabbit,$y) \wedge$ Plant $(\mathrm{y})$, we can use D1 and the unifier $\{x \mapsto$ rabbit, $y \mapsto c\}$ to obtain the subgoal $S_{1}$, which, together with D1, entails $S_{0}$. Then, we can use rule D2 and the unifier $\{y \mapsto c\}$ to obtain from $S_{1}$ the new subgoal $S_{2}$. The first conjunct in $S_{2}$ can be eliminated using the fact $\mathrm{A} 2$ in $\mathcal{D}^{e x}$ to produce $S_{3}$. From $S_{3}$ we can use again rule D1 to produce $S_{4}$. The first conjunct in $S_{4}$ can be eliminated using fact $\mathrm{A} 3$ in $\mathcal{D}^{e x}$ and finally we can obtain the empty goal by subsequently using fact A2 to eliminate the remaining atom. We have now shown that $\left\{\begin{array}{ll|l|l|l}D 1 & D 2 & A 2 & A 3\end{array}\right\} \models Q^{e x}$ (rabbit); therefore, facts A2 and A3 must be included in $\mathcal{D}_{f}$, and axioms T1 and T2 from $\mathcal{O}^{e x}$, from which rules D1 and D2 were (respectively) derived, must be included in $\mathcal{O}_{f}$.

To identify all the axioms and facts in $\mathcal{O} \cup \mathcal{D}$ that are relevant to $Q^{e x}$ (rabbit) and $Q^{e x}($ lion $)$, we need to consider all their possible backward chaining proofs. By doing so, we can show that only axioms T1 and T2, and facts A1, A2 and A3 are (possibly) relevant to determining the status of rabbit and lion.

\subsection{Technical Approach}

We start by formalising backward chaining proofs based on SLD-resolution.

Definition 1. A goal is a conjunction of function-free atoms $A_{1} \wedge \ldots \wedge A_{m}$. The SLD-resolution rule takes as premises a goal and a datalog rule, and it produces a new goal as follows

$$
\frac{A_{1} \wedge \ldots \wedge A_{m}, \quad C_{1} \wedge \ldots \wedge C_{q} \leftarrow B_{1} \wedge \ldots \wedge B_{p}}{A_{2} \theta \wedge \ldots \wedge A_{m} \theta \wedge B_{1} \theta \wedge \ldots, B_{p} \theta}
$$

where $\theta$ is the most general unifier of $A_{1}$ and $C_{j}$ for some $1 \leq j \leq q$. The new goal, together with the rule entail the original goal. An SLD-proof of a goal $G_{0}$ in a datalog program $\Gamma$ is a sequence of goals $G_{0} \stackrel{r_{1}, \theta_{1}}{\rightsquigarrow} G_{1} \rightsquigarrow \ldots \rightsquigarrow G_{n-1} \stackrel{r_{n}, \theta_{n}}{\rightsquigarrow} G_{n}$, where $G_{n}=\top$ and each $G_{i+1}$ is obtained from $G_{i}$ and rule $r_{i+1} \in \Gamma$ by means of a single $S L D$-resolution with substitution $\theta_{i+1}$. Finally, we say that a rule $r$ is relevant for $G_{0}$ in $\Gamma$ if there exists an $S L D$-proof of $G_{0}$ in $\Gamma$ involving $r$.

SLD-resolution is sound and complete for datalog: for each datalog program $\Gamma$, CQ $Q(\vec{x})=\exists \vec{y}(\varphi(\vec{x}, \vec{y}))$ and tuple of constants $\vec{a}$ we have $\Gamma \models Q(\vec{a})$ iff there exists an SLD-proof of the goal $\varphi(\vec{a}, \vec{y})$ in $\Gamma$. 
We are now ready to define the relevant fragments $\mathcal{O}_{f} \subseteq \mathcal{O}$ and $\mathcal{D}_{f} \subseteq \mathcal{D}$ that we can use to verify the answers in $G$ using an OWL reasoner.

Definition 2. Let $Q, \mathcal{O}$ and $\mathcal{D}$ be the input $C Q$, Horn ontology and dataset respectively, let $\Sigma_{U}$ be the upper bound datalog program for $\mathcal{O}$, and let $\Xi(\cdot)$ be the function mapping each axiom in $\mathcal{O}$ into its corresponding set of rules in $\Sigma_{U}$. Finally, let $G$ be the set of tuples between the lower and upper bounds. The $(Q, G)$-relevant fragments $\mathcal{O}_{f}$ of $\mathcal{O}$ and $\mathcal{D}_{f}$ of $\mathcal{D}$ are defined as follows $\mathcal{O}_{f}=\left\{\alpha \in \mathcal{O} \mid \exists \vec{a} \in G\right.$ and $\exists r \in \Xi(\alpha)$ s.t. $r$ is relevant for $Q(\vec{a})$ in $\left.\Sigma_{U} \cup \mathcal{D}\right\}$, $\mathcal{D}_{f}=\left\{\alpha \in \mathcal{D} \mid \exists \vec{a} \in G\right.$ s.t. $\alpha$ is relevant for $Q(\vec{a})$ in $\left.\Sigma_{U} \cup \mathcal{D}\right\}$.

The correctness of our approach is established by the following theorem.

Theorem 1. Let $\mathcal{O}_{f}$ and $\mathcal{D}_{f}$ be the $(Q, G)$-relevant fragments of $\mathcal{O}$ and $\mathcal{D}$, respectively. Then, $\mathcal{O} \cup \mathcal{D} \models Q(\vec{a})$ iff $\mathcal{O}_{f} \cup \mathcal{D}_{f}=Q(\vec{a})$ for each $\vec{a} \in G$.

The full proof of Theorem 1 can be found in our accompanying technical report 4 The idea behind it is, however, quite straightforward. The 'if' direction follows directly from the fact that $\mathcal{O}_{f} \cup \mathcal{D}_{f} \subseteq \mathcal{O} \cup \mathcal{D}$. For the 'only if' direction, assume that $\mathcal{O} \cup \mathcal{D} \models Q(\vec{a})$. From $\Sigma_{U} \models \mathcal{O}$ it follows that $\Sigma_{U} \cup \mathcal{D}=Q(\vec{a})$. By the completeness of SLD-resolution, there exists an SLD proof of $Q(\vec{a})$ in $\Sigma_{U} \cup \mathcal{D}$, and let $R$ be the set of rules used in this proof. By construction, the axioms in $\mathcal{O}$ and facts in $\mathcal{D}$ that correspond to rules in $R$ are contained in $\mathcal{O}_{f}$ and $\mathcal{D}_{f}$ respectively, and it can be further shown that these axioms entail $Q(\vec{a})$.

\subsection{An Optimised Backward Chaining Algorithm}

Computing all SLD-proofs for each answer in the gap between lower and upper bounds can be infeasible in practice with a naive backward chaining algorithm. Indeed, our problem is more challenging than typical backward chaining reasoning in datalog, where computing just a single proof suffices to verify the goal.

In this section, we describe an optimised algorithm for computing the set $R_{\vec{a}}$ of all rules that appear in an SLD proof of $Q(\vec{a})$ in $\Sigma_{U} \cup \mathcal{D}$, where the facts in $\mathcal{D}$ are treated as rules with an empty body, i.e., of the form $A(\vec{a}) \leftarrow$. To ensure termination of backward chaining, we apply the well-known tabling technique 24122. To improve performance, we use an aggressive pruning technique that exploits the upper and lower bound ontologies to detect irrelevant branches in the backward chaining tree. In the remainder of this section, we describe the specifics of our implementation of backward chaining.

Backward chaining with tabling. Our implementation of backward chaining with tabling is based on the techniques described in [22. We deviate from [22] in that our algorithm only terminates once all SLD-proofs of the goal are computed, and also in that we keep track of all rules used in such proofs.

We first describe our data structures. To keep track of all SLD proofs of a goal $Q(\vec{a})$ and all rules that occur in them, we maintain a labelled tree $t_{A}$ for each

\footnotetext{
4 http://tinyurl.com/bl5unv6
} 


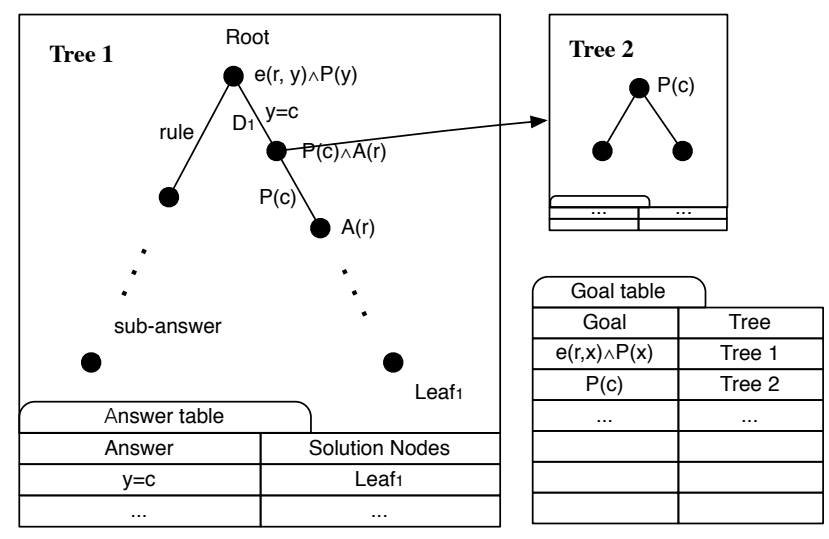

Fig. 2. Data Structure

encountered subgoal $A$ consisting of a single atom; additionally, we maintain a similar tree for the original goal $Q(\vec{a})$ (see Figure 2). The tree $t_{A}$ encodes all proofs of $A$; each node of $t_{A}$ is labelled with a goal and each edge of $t_{A}$ is labelled with a pair of a substitution and a datalog rule. The labels of each edge and the nodes that it connects encode an SLD resolution step, and a branch in $t_{A}$ encodes an SLD derivation. We associate with $t_{A}$ an answer table, which will eventually map each grounding of the root goal that proves it to the list of all relevant leaf nodes in $t_{A}$ for that grounding. We also maintain a global goal table mapping each relevant subgoal to its corresponding tree (c.f. Figure 2). Finally, we say that a node $u$ with associated goal $A_{1} \wedge \ldots \wedge A_{n}$ is linked to a tree $t$ if the first atom $A_{1}$ in the goal corresponds to the root of $t$ (e.g. node $D_{1}$ in Figure 2 is linked to Tree 2); we use the goal table to check whether $u$ is linked to $t$.

The backward chaining algorithm is initialised with a tree $t_{Q(\vec{a})}$ consisting of just a root node labelled with the goal $Q(\vec{a})$; then, we add an entry in the Goal table mapping $Q(\vec{a})$ to $t_{Q(\vec{a})}$ and we associate with $t_{Q(\vec{a})}$ an empty answer table. After this initialisation step, we evaluate the root node of $t_{Q(\vec{a})}$ using the recursive procedure described below, which takes as input a node $u$ in a tree $t$.

Case 1. If $u$ is labelled with $\mathrm{T}$, we have reached a proof of the goal $A(\vec{x})$ labelling the root of $t$. We take $A(\vec{a})$ to be the grounding of $A(\vec{x})$ using the composition of the substitutions on the path between the root of $t$ and the node $u$. We add $u$ to the list associated with $A(\vec{a})$ in the answer table of $t$. We then try to resolve $A(\vec{a})$ with the goal of every node that is linked to $t$. If we can resolve $A(\vec{a})$ with the goal of such a node $v$ using a substitution $\theta$, we add a child node $v^{\prime}$ to $v$, we label $v$ with the resolvent, and we label the edge between $v$ and $v^{\prime}$ with the pair $\langle A(\vec{a}), \theta\rangle$. We then recursively evaluate the node $v^{\prime}$.

Case 2. If $u$ is the root node of $t$, we resolve its goal with all possible rules, we create a child node for each of the resolvents, label the new nodes and edges accordingly, and recursively evaluate each of the child nodes.

Case 3. Otherwise, let $A_{1} \wedge \ldots \wedge A_{m}$ be the goal of $u$. 
3.1. If $A_{1}$ has not been tabled yet, i.e. it is not in the goal table, we initialise a tree $t^{\prime}$ with root node $v$, label $v$ with $A_{1}$, add an entry $\left\langle A_{1}, t^{\prime}\right\rangle$ to the goal table linking $u$ to $t$, and recursively process $v$;

3.2. otherwise, we retrieve the available answers for $A_{1}$ from its associated tree, resolve $u$ with those answers, create a child node for each of the resolvents, and recursively compute each added node.

Pruning. To improve performance, we apply a pruning technique that exploits the lower and upper bound ontologies. Let $v$ be a node with associated goal $Q_{v}:=A_{1} \wedge \ldots \wedge A_{m}$. Before recursively evaluating $v$, we proceed as follows.

- If $\operatorname{cert}\left(Q_{v}, \mathcal{O}_{U}, \mathcal{D}\right)=\emptyset$, we terminate the evaluation of the current node as this branch cannot lead to a proof. This is due to the fact that the rules used in the backward chaining algorithm are logically equivalent to $\mathcal{O}_{U} \cup \mathcal{D}$.

- If $Q_{v}$ contains no variables, and $\operatorname{cert}\left(Q_{v}, \mathcal{O}_{L}, \mathcal{D}\right) \neq \emptyset$, we create a child node for $v$, label it with $T$, and we label the new edge with the empty substitution and the set of atoms $A_{1}, \ldots, A_{m}$. We recursively evaluate the new node, after which we terminate the evaluation of $v$. We can do so because $\mathcal{O}_{L} \cup \mathcal{D}=Q_{v}$, and we know that the current branch will lead to exactly one proof.

- Otherwise, if $A_{1}$ contains no variable and $\operatorname{cert}\left(A_{1}, \mathcal{O}_{L}, \mathcal{D}\right) \neq \emptyset$, we create a child node for $v$, we label it with the goal $A_{2} \wedge \ldots \wedge A_{m}$, label the new edge with the empty substitution and the atom $A_{1}$, and recursively evaluate the new node, after which we terminate the evaluation of $v$. We can do so because $\mathcal{O}_{L} \cup \mathcal{D}=A_{1}$, and because $v$ has no other children.

Rule Extraction. The backward-chaining evaluation of the goal $Q(\vec{a})$ results in a forest of trees encoding all possible proofs of $Q(\vec{a})$ in $\Sigma_{U} \cup \mathcal{D}$. In addition to all proofs of $Q(\vec{a})$, the forest also contains many superfluous derivations that should be ignored. We now describe an algorithm that traverses the forest and extracts the set $R_{\vec{a}}$ of all rules that participate in proofs of $Q(\vec{a})$. The algorithm builds the set $R_{\vec{a}}$ by carrying out a bottom-up, breath-first search on the nodes in the forest whose goals appear in proofs of $Q(\vec{a})$. It proceeds as follows.

Step 1 Initialise a set $N$ with all solution nodes in the answer table of $t_{Q(\vec{a})}$.

Step 2 While $N$ is not empty, remove from $N$ a node $v$ with a goal $A_{1} \wedge \cdots \wedge A_{m}$. If $v$ has a parent, do the following:

2.1. Add the parent of $v$ to $N$.

2.2. If $v$ is a resolvent of its parent and a rule $r \in \Sigma_{U} \cup \mathcal{D}$, add $r$ to $R_{\vec{a}}$.

2.3. If $v$ is a resolvent of its parent and an answer $A$ from a tree $t$, retrieve all solution nodes for $A$ in $t$ and add them to $N$.

\section{Evaluation}

We have developed a prototype reasoner to carry out a preliminary evaluation. Our prototype integrates the RL reasoner RDFox $5^{5}$ and an OWL reasoner, which

\footnotetext{
5 http://www.cs.ox.ac.uk/isg/tools/RDFox/
} 
Table 1. Statistics for datasets

\begin{tabular}{|c|c|c|r|r|r|r|r|r|}
\hline Data & DL & Horn & Existential & Classes & Properties & Axioms & Individuals & Dataset \\
\hline LUBM $(\mathrm{n})$ & $\mathcal{S H I}$ & Yes & 8 & 43 & 32 & 93 & $1.7 \times 10^{4} n$ & $10^{5} n$ \\
\hline FLY & $\mathcal{S R \mathcal { I }}$ & Yes & 8,396 & 7,533 & 24 & 144,407 & 1,606 & 6,308 \\
\hline
\end{tabular}

Table 2. Results for LUBM(40)

\begin{tabular}{|c|c|c|c|c|c|c|c|c|c|c|}
\hline Query & $|V|$ & $n$ & $|G|$ & $\overline{t_{f}}$ & $\mid \overline{\mathcal{O}_{f} \mid}$ & $\left|\mathcal{D}_{f}\right|$ & \multicolumn{2}{|c|}{$t_{\text {check }}$} & \multicolumn{2}{|r|}{$t_{\text {total }}$} \\
\hline$M_{1}$ & 2 & 3 & 39 & 36.4 & 6 & 29041 & $\mathrm{H}:$ & 23.3 & $\mathrm{H}$ & 60.7 \\
\hline$M_{2}$ & 3 & 4 & 1 & 37.1 & 6 & 29004 & H: & 4.0 & $\mathrm{H}$ & 42.1 \\
\hline$M_{3}$ & 4 & 6 & 16 & 38.2 & 6 & 29054 & $\mathrm{H}:$ & 8.4 & $\mathrm{H}$ & 47.6 \\
\hline$M_{4}$ & 2 & 3 & 30 & 36.0 & 6 & 29032 & H: & 23.3 & $\mathrm{H}$ & 60.2 \\
\hline$M_{5}$ & 3 & 4 & 4 & 39.4 & 6 & 29010 & H: & 24.0 & $\mathrm{H}$ & 64.3 \\
\hline$M_{6}$ & 4 & 6 & 29 & $2,845.8$ & 10 & 87209 & $\mathrm{H}:$ & 483.0 & $\mathrm{H}$ & 3339.4 \\
\hline$M_{7}$ & 3 & 5 & 15 & 38.0 & 6 & 29033 & H: & 10.3 & $\mathrm{H}$ & 49.3 \\
\hline$M_{8}$ & 3 & 5 & 14 & 39.3 & 6 & 29038 & H: & 11.9 & $\mathrm{H}$ & 52.2 \\
\hline$M_{9}$ & 3 & 4 & 10 & 328.9 & 12 & 86785 & H: & 556.2 & $\mathrm{H}$ & 886.7 \\
\hline$S$ & 1 & 2 & 39 & 310.0 & 12 & 86802 & $\begin{array}{l}\mathrm{H}: \\
\mathrm{P}:\end{array}$ & $\begin{array}{r}1,780.0 \\
16,592.1\end{array}$ & & $\begin{array}{r}2,126.5 \\
16,870.0\end{array}$ \\
\hline
\end{tabular}

in our case can be either HermiT[16] or Pellet 23. RDFox is used to compute lower and upper bound query answers (c.f. Step 1 in Section 3), as well as to assist with pruning during backward chaining (c.f. Section 5.3). The OWL reasoner is used with the fragments computed by the backward chaining algorithm (c.f. Step 3 in Section 3 to determine the status of any tuples in the gap.

RDFox is a in-memory triple store that supports OWL 2 RL and datalog reasoning, and uses shared memory parallel reasoning for increased efficiency and scalability. An important feature of RDFox is its rapid query response time - this is particularly relevant during backward chaining, where queries are used in a crucial pruning optimisation. HermiT and Pellet are well-established OWL reasoners that provide support for CQ answering. HermiT can answer tree-shaped CQs with a single answer variable; Pellet supports SPARQL CQs.

In our experiments we have used LUBM and the Fly Anatomy ontology as test sets; their key features are summarised in Table 1 . All tests were performed on a 14 core $3.30 \mathrm{GHz}$ Intel Xeon E5-2643 with $125 \mathrm{~GB}$ of RAM, and running Linux 2.6.32. All times are given in seconds.

Evaluation results for LUBM. LUBM is a well-known benchmark ontology that comes with a predefined dataset generator parameterised by the number of universities. We tested our reasoner on $\operatorname{LUBM}(40)$, which contains in its dataset over 4 million facts about 40 universities. We used the 14 standard LUBM queries as well as 78 synthetic queries generated using SyGENiA [12.

Using RDFox, we were able to compute lower and upper bound answers for all 92 queries in less than 20s (c.f. Step 1 in Section 3). As the focus of this paper is on checking the tuples in the gap between the two bounds of a given query, we concentrate our attention on those queries whose bounds do not 
Table 3. Results for FLY

\begin{tabular}{|c|r|r|r|r|r|r|r|r|r|}
\hline Query & $|V|$ & \multicolumn{1}{|c|}{$n$} & \multicolumn{1}{|c|}{$|G|$} & \multicolumn{1}{c|}{$t_{f}$} & \multicolumn{1}{|c|}{$\left|\mathcal{O}_{f}\right|$} & \multicolumn{1}{|c|}{$\left|\mathcal{D}_{f}\right|$} & $t_{\text {check }}(\mathrm{H})$ & $t_{\text {total }}$ & \multicolumn{1}{c|}{$t_{\text {HermiT }}$} \\
\hline$Q_{1}$ & 2 & 3 & 803 & 108.9 & 224 & 4515 & 45.9 & 155.2 & $3,465.9$ \\
\hline$Q_{2}$ & 3 & 5 & 342 & 97.7 & 224 & 4054 & 16.0 & 114.0 & $3,179.0$ \\
\hline$Q_{3}$ & 1 & 1 & 28 & 91.0 & 217 & 3712 & 0.9 & 92.3 & $5,863.3$ \\
\hline$Q_{4}$ & 2 & 3 & 25 & 94.3 & 233 & 3762 & 4.7 & 99.2 & $2,944.3$ \\
\hline$Q_{5}$ & 2 & 2 & 518 & 100.3 & 222 & 3712 & 24.0 & 124.6 & $3,243.7$ \\
\hline
\end{tabular}

coincide. Only 6 queries show a non-empty gap $\left(Q_{3}, Q_{45}, Q_{51}, Q_{64}, Q_{67}, Q_{69}\right)$ all of them SyGENiA generated - and, due to the relatively simple nature of the LUBM ontology, it is inevitable that these queries look very similar. Since the generated queries tend to produce unrealistically large answers, we added some additional terms to those queries in order to make them more specific and thus return smaller answers. The resulting 10 "non-trivial" queries, denoted by $M_{1}-M_{9}$ and $S$, are all tree-shaped CQs, with $S$ being the only SPARQL CQ $\sqrt{6}$ Performance on these queries is summarised in Table 2, where $|V|, n$ and $|G|$ denote the number of variables, the number of triple patterns and the number of gap tuples for each query respectively; $t_{f},\left|\mathcal{O}_{f}\right|$ and $\left|\mathcal{D}_{f}\right|$ denote the time needed to compute the relevant fragments $\mathcal{O}_{f}$ and $\mathcal{D}_{f}$ using backward chaining, and their respective sizes; $t_{\text {check }}$ denotes the total time required for checking all the tuples in $G$ using the OWL reasoner (c.f. Step 3 in Section 3); and $t_{\text {total }}$ denotes the total time for answering the query. We have presented timings for Hermit $(\mathrm{H})$ on all queries, and Pellet $(\mathrm{P})$ on the single SPARQL CQ.

We can observe that backward chaining times were rather modest for all queries but $M_{6}$, for which the computed backward chaining tree had a very large branching factor. We can also observe that for all queries the dataset fragment $\mathcal{D}_{f}$ contains only about $2 \%$ of the facts in $\operatorname{LUBM}(40)$, while $\mathcal{O}_{f}$ contained just a few schema-level axioms. This significant reduction in size made it possible for HermiT to verify answer tuples in reasonable time; indeed, query answering for $\operatorname{LUBM}(40)$ is far beyond the capabilities of HermiT or Pellet, and we were unable to verify even a single answer tuple using either OWL reasoner over the original ontology and dataset. A standard optimisation applied by RL reasoners such as OWLim is to classify the original ontology first and add the entailed subsumption axioms in $\mathcal{O}_{L}$. Although this optimisation closes the gap between the lower and upper bounds for query $S$, allowing, for example, OWLim to compute all answers for $S$, it has no effect on queries $M_{1}-M_{9}$.

To test the scalability of our reasoner, we have also evaluated queries $M_{1}$, $M_{6}, M_{9}$ and $S$ against the datasets LUBM(1)-LUBM(40) (results for queries $M_{2}-M_{5}, M_{7}$ and $M_{8}$ are similar to those for $\left.M_{1}\right)$. The results of the evaluation are summarised in Figure 3, which shows the timings and memory usage of our reasoner for the different datasets.

Evaluation results for FLY. Fly Anatomy is a realistic and complex ontology describing the anatomy of flies, which comes with a dataset containing more

\footnotetext{
${ }^{6}$ All test queries are available at http://tinyurl.com/ccmwvc6.
} 


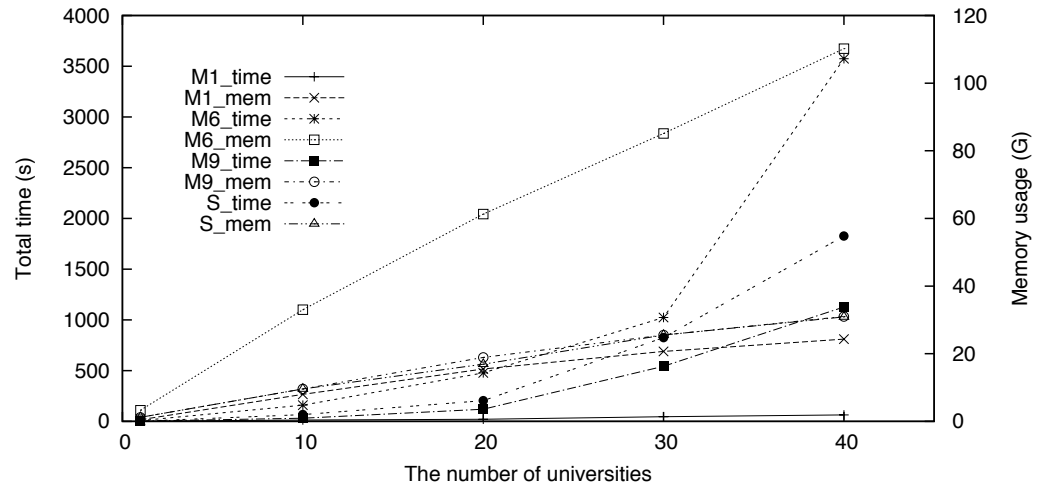

Fig. 3. Scalability test for LUBM(1)-LUBM(40)

than 6,000 facts. We have tested our system using 5 realistic queries provided by the domain experts who are developing the ontology; all are CQs with nondistinguished variables, so we were only able to use HermiT in the evaluation.

RDFox was able to compute lower and upper bound answers for all 5 queries in less than 15s, with the bounds being different in all cases. Our results are summarised in Table 3, columns 1-9 are the same as in Table 2, and column 10 gives the time taken for HermiT to answer the query - in contrast to the case of $\operatorname{LUBM}(40)$, HermiT is able to answer all these queries directly. In each case, $\mathcal{O}_{f}$ contained less than $0.2 \%$ of all the schema-level axioms; in contrast, $\mathcal{D}_{f}$ contained about $60 \%$ of the facts in the dataset. The reduction in number of schema-level axioms had a significant effect on performance: our reasoner took less than 200s per query, whereas HermiT required more than 3,000s per query.

\section{Related Work}

In recent years there has been a growing interest in the problem of query answering over ontologies and large-scale datasets. Some OWL 2 reasoners, such as HermiT, Pellet and RACER, support query answering, but despite intensive efforts at optimisation they can only deal with modestly-sized datasets [14[15]10.

The idea of using a rule-based engine for query answering over ontologies in the description logic $\mathcal{S H I} \mathcal{I}$ was proposed by Hustadt et al. [11 and implemented in the KAON2 system. The transformation of the ontology, however, results in a disjunctive datalog program which is exponential in the worst case. An alternative approach based on tableaux reasoning and data summarisation was implemented in the reasoner SHER, which is complete for the description logic

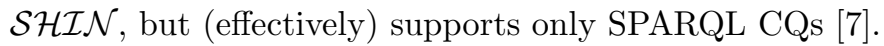

Many specialised query answering techniques have been developed for ontologies in the QL and RL profiles of OWL 2. RL reasoners such as OWLim, Ora- 
cle's and RDFox are based on forward-chaining reasoning. QL reasoners such as QuOnto [1, Presto [21, and Quest [20] are based on query rewriting. These reasoners are, however, incomplete for ontologies outside the relevant profile. Query rewriting techniques have been extended to more expressive Horn Description Logics, and implemented in systems such as REQUIEM [19] and Clipper [8.

The idea of combining a profile-specific reasoner with a fully-fledged OWL 2 reasoner was proposed in 2, but only for ontology classification. The idea of transforming the ontology, data, and/or query to obtain upper bound query answers has also received some attention in the Semantic Web literature. In addition to our own previous work [27, approximations into OWL 2 QL [13]18] and into Datalog 25] have also been explored; however, all these techniques are worst case exponential, and the question of how to deal with cases where upper and lower bounds do not coincide was not considered.

\section{Conclusion}

In this paper we have described a hybrid approach for complete query answering over Horn OWL 2 ontologies. Our technique combines a scalable OWL 2 RL reasoner with a fully-fledged OWL 2 reasoner s.t. most of the computational workload is delegated to the RL reasoner, with the OWL 2 reasoner being used only as necessary to ensure completeness. We have implemented a prototype reasoner that integrates the RL reasoner RDFox and the OWL 2 reasoner HermiT. A preliminary evaluation of our prototype produced very promising results: we managed to compute in reasonable time the exact answers to a range of queries over LUBM(40) - results that are far beyond the capabilities of any other OWL 2 reasoner known to us. Our system also outperforms HermiT on the realistic Fly ontology by at least an order of magnitude. We are currently working on an extension to support query answering over arbitrary OWL 2 ontologies (and not just Horn ontologies), as well as on several promising optimisations.

Acknowledgements. The research was supported by the EPSRC funded ExODA and Score! projects, the Royal Society, and the EU FP7 Optique project.

\section{References}

1. Acciarri, A., Calvanese, D., Giacomo, G.D., Lembo, D., Lenzerini, M., Palmieri, M., Rosati, R.: Quonto: Querying ontologies. In: AAAI. pp. 1670-1671 (2005)

2. Armas Romero, A., Cuenca Grau, B., Horrocks, I.: MORe: Modular combination of owl reasoners for ontology classification. In: ISWC. pp. 1-16 (2012)

3. Bishop, B., Kiryakov, A., Ognyanoff, D., Peikov, I., Tashev, Z., Velkov, R.: OWLim: A family of scalable semantic repositories. Semantic Web J. 2(1), 33-42 (2011)

4. Bry, F., Eisinger, N., Eiter, T., Furche, T., Gottlob, G., Ley, C., Linse, B., Pichler, R., Wei, F.: Foundations of rule-based query answering. In: RR. pp. 1-153 (2007)

5. Cali, A., Gottlob, G., Lukasiewicz, T., Marnette, B., Pieris, A.: Datalog+/-: A family of logical knowledge representation and query languages for new applications. In: LICS (2010) 
6. Cuenca Grau, B., Horrocks, I., Krötzsch, M., Kupke, C., Magka, D., Motik, B., Wang, Z.: Acyclicity conditions and their application to query answering in description logics. In: Proc. of the 13th Int. Conf. on Principles of Knowledge Representation and Reasoning (KR 2012) (2012), download/2012/CHKKMMW12a.pdf

7. Dolby, J., Fokoue, A., Kalyanpur, A., Ma, L., Schonberg, E., Srinivas, K., Sun, X.: Scalable grounded conjunctive query evaluation over large and expressive knowledge bases. In: The Semantic Web-ISWC 2008, pp. 403-418. Springer (2008)

8. Eiter, T., Ortiz, M., Šimkus, M., Tran, T.K., Xiao, G.: Query rewriting for hornshiq plus rules. In: AAAI (2012)

9. Haarslev, V., Möller, R.: RACER system description. J. of Automated Reasoning (JAR) pp. 701-705 (2001)

10. Haarslev, V., Hidde, K., Möller, R., Wessel, M.: The RacerPro knowledge representation and reasoning system. Semantic Web 3(3), 267-277 (2012)

11. Hustadt, U., Motik, B., Sattler, U.: Reasoning in Description Logics by a Reduction to Disjunctive Datalog. Journal of Automated Reasoning 39(3), 351-384 (2007)

12. Imprialou, M., Stoilos, G., Grau, B.: Benchmarking ontology-based query rewriting systems. In: Proceedings of the Twenty-Sixth AAAI Conference on Artificial Intelligence, AAAI (2012)

13. Kaplunova, A., Möller, R., Wandelt, S., Wessel, M.: Towards scalable instance retrieval over ontologies. Knowledge Science, Engineering and Management (2010)

14. Kollia, I., Glimm, B.: Cost based query ordering over OWL ontologies. In: ISWC. pp. 231-246 (2012)

15. Kollia, I., Glimm, B., Horrocks, I.: SPARQL query answering over OWL ontologies. In: ESWC. pp. 382-396 (2011)

16. Motik, B., Shearer, R., Horrocks, I.: Hypertableau reasoning for description logics. J. of Artificial Intelligence Research (JAIR) 36(1), 165-228 (2009)

17. Motik, B., Cuenca Grau, B., Horrocks, I., Wu, Z., Fokoue, A., Lutz, C.: OWL 2 Web Ontology Language Profiles (2nd Edition) (2012), W3C Recommendation.

18. Pan, J., Thomas, E., Zhao, Y.: Completeness guaranteed approximations for OWLDL query answering. DL 477 (2009)

19. Pérez-Urbina, H., Horrocks, I., Motik, B.: Efficient query answering for OWL 2. In: ISWC. pp. 489-504 (2009)

20. Rodriguez-Muro, M., Calvanese, D.: High performance query answering over DLLite ontologies. In: KR (2012)

21. Rosati, R.: Prexto: Query rewriting under extensional constraints in DL-Lite. In: ESWC. pp. 360-374 (2012)

22. Sagonas, K., Swift, T.: An abstract machine for tabled execution of fixed-order stratified logic programs. ACM Transactions on Programming Languages and Systems (TOPLAS) 20(3), 586-634 (1998)

23. Sirin, E., Parsia, B., Cuenca Grau, B., Kalyanpur, A., Katz, Y.: Pellet: A practical OWL-DL reasoner. J. Web Semantics (JWS) 5(2), 51-53 (2007)

24. Tamaki, H., Sato, T.: OLD resolution with tabulation. In: ICLP. pp. 84-98 (1986)

25. Tserendorj, T., Rudolph, S., Krötzsch, M., Hitzler, P.: Approximate OWLreasoning with screech. In: RR. pp. 165-180 (2008)

26. Wu, Z., Eadon, G., Das, S., Chong, E.I., Kolovski, V., Annamalai, M., Srinivasan, J.: Implementing an inference engine for RDFS/OWL constructs and user-defined rules in Oracle. In: ICDE. pp. 1239-1248 (2008)

27. Zhou, Y., Cuenca Grau, B., Horrocks, I., Wu, Z., Banerjee, J.: Making the Most of your Triple Store: Query Answering in OWL 2 Using an RL Reasoner. In: WWW (2013) 